\title{
Odorant G protein-coupled receptors as potential therapeutic targets for adult diffuse gliomas: a systematic analysis and review
}

\author{
Hee Jin Cho ${ }^{1,2, *}$ E JaeHyung $\mathrm{Koo}^{3,4,5, *}$ \\ ${ }^{1}$ Department of Biomedical Convergence Science and Technology, Kyungpook National University, Daegu 41566, ${ }^{2}$ Cell and Matrix \\ Research Institute, Kyungpook National University, Daegu 41944, ${ }^{3}$ Department of New Biology, DGIST, Daegu 42988, ${ }^{4}$ New Biology \\ Research Center (NBRC), DGIST, Daegu 42988, ${ }^{5}$ Korea Brain Research Institute (KBRI), Daegu 41062, Korea
}

\begin{abstract}
Odorant receptors (ORs) account for about $60 \%$ of all human G protein-coupled receptors (GPCRs). OR expression outside of the nose has functions distinct from odor perception, and may contribute to the pathogenesis of disorders including brain diseases and cancers. Glioma is the most common adult malignant brain tumor and requires novel therapeutic strategies to improve clinical outcomes. Here, we outlined the expression of brain ORs and investigated OR expression levels in glioma. Although most ORs were not ubiquitously expressed in gliomas, a subset of ORs displayed glioma subtype-specific expression. Moreover, through systematic survival analysis on OR genes, OR51E1 (mouse Olfr558) was identified as a potential biomarker of unfavorable overall survival, and OR2C1 (mouse Olfr15) was identified as a potential biomarker of favorable overall survival in isocitrate dehydrogenase (IDH) wild-type glioma. In addition to transcriptomic analysis, mutational profiles revealed that somatic mutations in OR genes were detected in $>60 \%$ of glioma samples. OR5D18 (mouse Olfr1155) was the most frequently mutated OR gene, and OR5AR1 (mouse Olfr1019) showed IDH wild-type-specific mutation. Based on this systematic analysis and review of the genomic and transcriptomic profiles of ORs in glioma, we suggest that ORs are potential biomarkers and therapeutic targets for glioma. [BMB Reports 2021; 54(12): 601-607]
\end{abstract}

\section{INTRODUCTION}

Adult diffuse gliomas account for about $80 \%$ of primary malignant tumors in brain. Glioblastoma, which is also referred to as WHO grade IV glioma, is one of the most lethal cancers and is associated with only 15 months median survival (1-3).

*Corresponding authors. Hee Jin Cho, Tel: +82-53-950-4549; Fax: +82-508-902-2674; E-mail: heejincho@knu.ac.kr; JaeHyung Koo, Tel: +82-53-785-6112; Fax: +82-53-785-1819; E-mail: jkoo001@dgist.ac.kr

https://doi.org/10.5483/BMBRep.2021.54.12.165

Received 17 November 2021, Revised 22 November 2021, Accepted 22 November 2021

Keywords: Glioma, GPCR, Odorant receptor, OR51E1, OR51E2
According to the recent $2021 \mathrm{WHO}$ classification, adult diffuse gliomas can be classified into three subtypes: isocitrate dehydrogenase (IDH)-mutant (typically, astrocytoma), IDH mutant with chromosome $1 p$ and $19 q$ co-deletion (typically, oligodendroglioma), and IDH-wildtype (typically, glioblastoma) (4). These three subtypes exhibit distinct genomic features and clinical outcomes. Glioma was at the forefront of the WHO genomic characterization and is one of the most extensively studied cancers (5-7). Yet despite extensive studies on gliomas, new therapeutic strategies are needed to improve clinical outcomes. Exploring as yet under-studied potential drug targets may be key in providing therapeutic options for this disease.

The family of $\mathrm{G}$ protein-coupled receptors (GPCRs) is the largest one of transmembrane proteins. They are the largest protein family of druggable targets for approved drugs; about 500-700 drugs that target GPCRs are approved for clinical use $(8,9)$. Repurposing approved GPCR-targeting drugs is an efficient way of developing new treatment options, as it reduces the time and effort involved in this process. To develop new treatment options for cancer, it is essential to carry out a systematic and intensive analysis of the roles of GPCRs in cancer. Among GPCRs, chemokine receptors, such as $\mathrm{C}-\mathrm{X}-\mathrm{C}$ motif chemokine receptor 4 (CXCR4), have been widely studied in gliomas $(10,11)$. However, in non-olfactory tissues, the role of odorant receptors (ORs), which are the largest subfamily of GPCRs and account for about $60 \%$ of all human GPCRs, remains unknown (12-14). In particular, the role of ORs in cancers such as glioma is largely unexplored, despite the enormous number of OR genes (13, 15). A well-organized review of non-olfactory GPCRs as therapeutic targets in glioblastoma by Byrne et al. was recently published (16). However, this review was not able to evaluate the potential of ORs as therapeutic targets in glioma due to the limited number of published studies. Here, we have conducted a systematic analysis and review to investigate the potential of ORs as therapeutic targets in glioma.

\section{MAIN TEXT}

Classification of HUGO-registered GPCR genes

Human GPCR families have been assigned 1414 unique gene symbols, including pseudogenes, by the HUGO (Human Genome

ISSN: 1976-670X (electronic edition)

Copyright (C) 2021 by the The Korean Society for Biochemistry and Molecular Biology

(c) This is an open-access article distributed under the terms of the Creative Commons Attribution Non-Commercial License (http://creativecommons.org/licenses/by-nc/4.0) which permits unrestricted non-commercial use, distribution, and reproduction in any medium, provided the original work is properly cited. 
Organization) Gene Nomenclature Committee (17). GPCRs are transmembrane receptors that bind specific ligands and activate intracellular signaling, thereby mediating senses such as vision, smell, and taste $(18,19)$. There are diverse subgroups within GPCR superfamilies. Human GPCRs can be largely classified into five families based on phylogenetic grouping, namely glutamate, rhodopsin, adhesion, frizzled, and secretin receptors; this grouping is known as the GRAFS classification $(20,21)$. In particular, the rhodopsin-like class comprises 93\% (1315/1414) of human GPCRs, and ORs make up the largest portion, $61.7 \%$ (873/1414), of rhodopsin-like GPCRs according to the HUGO Gene Nomenclature Committee (17).

\section{OR expressions of brain in normal and diseases models}

A previous transcriptome analysis of GPCRs revealed that the expression levels of GPCRs vary by tissue depending on the physiological function of the GPCR (22). ORs are expressed in olfactory epithelium where they conduct their major function of sensing molecules related to smell. In addition, expression of ORs is detected outside of the nose; these receptors are called "ectopic OR" $(13,14,23)$. In particular, expression of the limited number of ORs in brain was detected using PCR and in situ hybridization techniques (24-27), and recently, systematic analysis on OR expression using microarray or RNAsequencing revealed that various ORs such as OR51E1/OIfr558 and OR51E2/Olfr78 were expressed in brain region including cerebellum (28-30). In addition to expression of intact OR gene, Flegel et al. also discovered the chimeric transcript (OR2W3/ Olfr322-Trim58) and internal splice variant (OR2L13/Olfr166) of ORs in brain (29). Moreover, altered gene expression levels of ORs were reported in brain diseases such as Alzheimer's disease (AD) and Parkinson's disease (PD); up-regulation of Olfr110/ 111 (human OR5V1) in aging and AD models $(31,32)$, downregulation of Olfr1494/OR10Q1, Olfr1324, Olfr1241/OR4A16, and Olfr979/OR10G9 in central nervous system injury (33), down-regulation of OR2L13/Olfr166, OR1E1, OR2J3, OR52L1/ Olfr685, and OR11H1 in PD (34), and down-regulation of OR52L1/Olfr685, OR51E1/Olfr558, OR2T1/Olfr31, OR2T33, OR52H1/Olfr648, OR2D2/Olfr715 and OR10G8 in schizophrenia (35). The expression of Olfr316/OR2AK2, Olfr558/OR51E1, Olfr166/OR2L13, Olfr287/OR10AD1, Olfr883, Olfr1344 and Olfr1505/OR9I1 were confirmed in the mesencephalic dopaminergic neurons whose abnormal functions are related to PD and schizophrenia as well (36). We also reported the expression (Olfr110/OR5V1, Olfr111/OR5V1, Olfr920, Olfr1417, and Olfr99/ OR1G1) and interaction of brain OR with its ligand in brain cells, such as astrocytes and microglia (37-39). Together, Ferrer et al. emphasized the need to study the role of ORs in mammalian brain; this assertion was based on a summary of studies of ORs in human and mouse brain and the altered OR gene expression levels on neurodegenerative diseases (40).

\section{Publications related to GPCRs in glioma}

Next-generation sequencing technology has been widely used in cancer research, including systematic genomic and transcriptomic studies of GPCRs to identify new potential therapeutic targets in cancer (41-44). Until recently, GPCR-based genomic studies focused on non-olfactory receptors, but newer systematic studies highlight the role of ORs as potential contributors to tumorigenesis (45-48).

Given that ORs may have potential oncogenic roles in solid tumors, and that ORs expressed in normal brain and during neurodegenerative disease have functions distinct from odor perception, we hypothesized that ectopic ORs would perform tumor-related functions in brain. As further evidence for the role of ectopic ORs in tumor-related functions in brain, we previously reported that Olfr78 (human homolog, OR51E2) induced M2 polarization, thereby promoting tumor progression and metastasis (49). M2-like tumor-associated macrophages are abundant in the glioma microenvironment, and mediate increased resistance to radiotherapy and immunotherapy (50-52). Glioma is the most common brain malignant tumor. Therefore, a large number of studies have investigated, including the role of GPCRs in glioma. Our current analysis of PubMed literature showed that the oncogenic or tumor-suppressive roles of nonolfactory GPCRs, including CXCR4, SMO, and DRD2, have been reported in over 1,000 publications (Fig. 1A). However, only three publications describing the glioma-related functions of ORs were found: 1) Glioblastoma patients harboring OR4Q3 (mouse Olfr735) mutations have a poor prognosis (53); 2) OR51F2/ Olfr568 is a potential mediator of the 4-gene signature predicting temozolomide response in lower-grade glioma (LGG) patients (54); 3) OR7E156P acts as a long non-coding RNA that contributes to tumor growth and invasion via the OR7E156P/miR-143/ HIF1A axis in glioma cells (55). Also, recent single-cell transcriptome study of ORs in pan-cancer revealed that $65.9 \%$ and $55.2 \%$ of glioblastoma and astrocytoma cells express OR genes, obeying "one neuron-one receptor" rule (i.e., a single neuron express one OR gene $(56,57))$ although this study was not searchable in PubMed by the query "(OR gene name [Text Word]) AND ((glioma[Text Word]) OR (glioblastoma[Text Word])" (45).

\section{RNA expression of GPCRs in glioma}

To investigate the overall expression pattern of GPCRs in glioma, we used the integrated RNA-seq-based expression profiles of LGG and glioblastoma multiforme (GBM) from the Cancer Genome Atlas (TCGA; downloaded from https://gdac.broadinsti tute.org) (6). A total of 656 primary LGG and GBM samples were available, of which 229, 258, and 169 samples were IDH wild-type (IDHwt), IDH mutant without chr1p/chr19q co-deletion (IDHmut-non-codel), and IDH mutant with chr1p/chr19q co-deletion (IDHmut-codel), respectively. In total, 721 GPCR genes were mapped on the integrated LGG and GBM expression profiles, and 375 of 721 genes were categorized as OR genes, including 11 pseudogenes. RNA expression profiles of glioma revealed that $60 \%$ of non-olfactory GPCR genes show ubiquitous expression (expressed $\left(\log _{2}(\operatorname{RSEM}+1)>0.1\right)$ in $>90 \%$ 

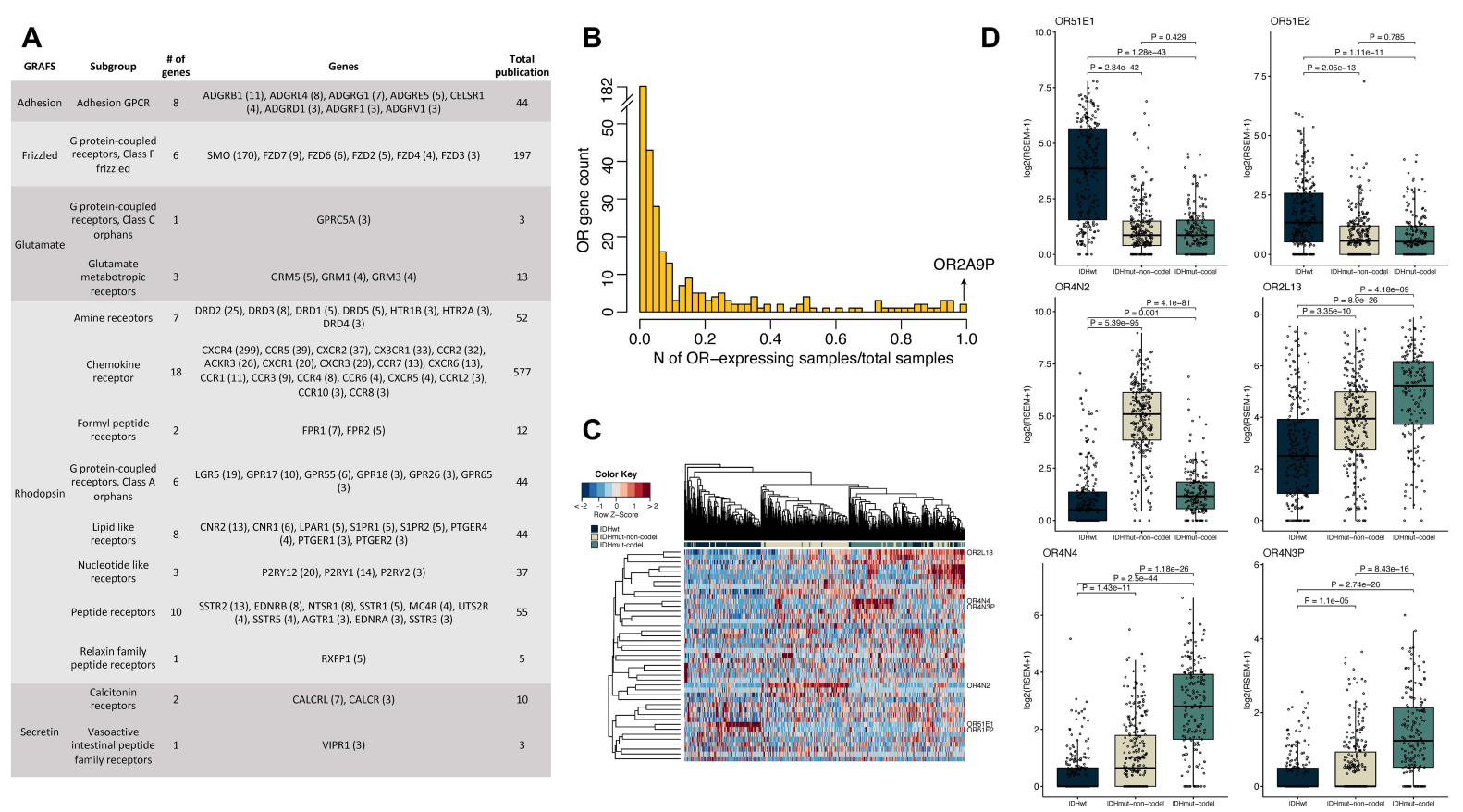

Fig. 1. GPCR-related publications and the distribution of odorant receptor (OR) gene expression in glioma from the Cancer Genome Atlas. (A) A table describing GPCR genes associated with gliomas in more than two publications. The number in parenthesis represents the number of publications that was found in PubMed. (B) A bar plot showing the OR-expressing sample ratio for each OR gene. The x-axis represents the glioma sample ratio, which is defined by the number of OR-expressing samples divided by the total sample number, and the $y$-axis represents the number of ORs. (C) A heatmap showing unsupervised clustering of ORs that have highly variable expression levels between samples (standard deviation $\geq 0.3$ and expressed in $>33.3 \%$ of the total number of samples). (D) A box plot showing OR gene expression levels between the three glioma subtypes. The P-values were calculated from the Student's t-test. A black dot represents one sample.

of samples, and $81 \%$ of the non-olfactory GPCR genes were expressed in more than half of TCGA gliomas. Three-quarters of ORs were barely expressed, and the remainder of ORs were detected only in a subset of gliomas (Fig. 1B). These observations imply that ORs might exhibit sample- or subtype-specific functions in glioma and the glioma microenvironment. Hence, we investigated the expression and mutational profiles of ORs by the glioma subtype and their clinical relevance.

\section{Expression of ORs in glioma}

When we explored the expression profiles of ORs in more detail, 373 of 375 (99.5\%) ORs mapped on the integrated LGG and GBM expression profiles were expressed in at least one sample (Fig. 1B). However, 237 ORs (63.5\%) were expressed only in a small subset $(<5 \%)$ of glioma samples. On the other hand, expression of OR2A9P was detected in all gliomas, and another eight ORs (OR7D2, OR2H2/Olfr90, OR7A5/Olfr57, OR2A7, OR13A1/Olfr211, OR2L13/Olfr166, OR2W3/Olfr322, and OR2C1/Olfr15) were expressed in $>90 \%$ of glioma samples.

Because glioma subtypes have subtype-specific genomic and clinical features, we performed unsupervised hierarchical clustering using 43 ORs with highly variable expression between glioma samples (standard deviation $\geq 0.3$ and expressed in more than one-third of samples) to identify whether the different subtypes of gliomas displayed distinct OR expression profiles (6). Interestingly, each subtype was clustered together, indicating that enrichment of the different subsets of ORs depended on the glioma subtype (Fig. 1C). High expression levels of OR51E1 (mouse Olfr558) and OR51E2 (mouse Olfr78) were noticeable in IDHwt subtypes; OR4N2 (mouse Olfr733) was expressed in IDHmut-non-codel subtypes; and expression of OR2L13 (mouse Olfr166), OR4N4, and OR4N3P was higher in IDHmut-codel subtypes than in other subtypes (Fig. 1C, D). Similar to our findings, Pappula et al. identified the 4637 differentially expressed genes (DEGs) between IDH1-mutant and IDH1-wild-type astrocytoma patients, and 18 OR genes including OR4N2 (mouse Olfr733) belonged to the DEGs (58). The distinct expression of ORs in the three glioma subtypes implies that ORs might participate in tumor formation and in the construction of the tumor microenvironment during gliomagenesis.

\section{Clinical relevance of OR expression in glioma}

Next, we performed Cox regression survival analysis using gene expression levels to investigate the prognostic potential of OR gene expression. Univariate Cox regression analysis revealed that seven genes (OR2AE1, OR2B6/Olfr11, OR10AD1/Olfr287, 
OR51E1/Olfr558, OR7C1/Olfr1352, OR2A9P, and OR51E2/Olfr78) were associated with unfavorable overall survival (adjusted P-value $<0.05$ and hazard ratio $>1$ ). There were 18 genes associated with favorable overall survival, including OR4N2 (mouse Olfr733), OR4N4, and OR4N3P (adjusted P-value $<0.05$ and hazard ratio $<1$ ) (Fig. 2A). However, all of the survival-associated genes were filtered out during multivariate analysis with age, IDH status, and histologic grade; these factors are known prognostic markers of gliomas (59). Therefore, we re-conducted a survival analysis for each subtype. OR51E1 (mouse Olfr558, adjusted $\mathrm{P}$-value $=0.03$; hazard ratio $=1.15$ ) and OR2C1 (mouse Olfr15, adjusted P-value $=0.03$; hazard ratio $=0.68$ ) were associated with potential unfavorable and favorable outcomes, respectively for IDHwt glioma (Fig. 2B-D). OR51E1 (mouse Olfr558) was previously reported as a potential biomarker for prostate cancer and small intestine neuroendocrine carcinoma (60). Four genes (OR13J1/Olfr71, OR2B6/Olfr11, OR4K2/Olfr730, and OR7A5/Olfr57) were associated with poor overall survival in IDHmut-non-codel gliomas, and another four genes (OR2H2/ Olfr90, OR5K2/Olfr177: favorable; OR51E1/Olfr588, OR7C1/
Olfr1352: unfavorable) were associated with overall survival in IDHmut-codel gliomas. However, no OR genes were associated with a significant prognosis in the IDHmut-non-codel and IDHmutcodel subtypes after P-value adjustment (Fig. 2B). OR2B6 (mouse Olfr11) has also been previously identified as a cancer biomarker (48); thus further analysis of these identified survival-associated OR genes is needed to determine if they are potential biomarkers and/or therapeutic targets in glioma $(46,48)$.

\section{Mutational profiling of ORs in glioma}

Single-nucleotide variants (SNV) and small insertion/deletions (INDEL) can cause changes in protein structure or mRNA degradation through nonsense-mediated mRNA decay; thus, SNV and small INDEL can generate proteins with gain or loss of functions. According to previous studies $(61,62)$, various natural variants of human ORs exist. Thus, in addition to transcriptomic analysis, we explored the mutational status of ORs in gliomas. Among 799 primary glioma samples that were subjected to either whole-exome or whole-genome sequencing from TCGA, 499 samples carried at least one OR non-silent
A

\begin{tabular}{|c|c|c|c|c|c|}
\hline Gene & Outcome & Hazard ratio & P-value & $\begin{array}{c}\text { Adjusted } \\
\text { P-value }\end{array}$ & $\begin{array}{l}\text { Multivariate } \\
\text { P-value }\end{array}$ \\
\hline ORZAE1 & Unfavorable & 1.810 & 1.0. E- -05 & & \\
\hline OR2B6 & Unfavorable & 1.635 & 1.8.E- -99 & 1.1.E-E -8 & 0.37 \\
\hline OR10AD1 & Unfavorable & 1.547 & 2.0.E-03 & 4.3.E-03 & 0.50 \\
\hline OR51E1 & Unfavorable & 1.493 & 1.5.E- -32 & 6.4.E-31 & 0.36 \\
\hline OR7C1 & Unfavorable & 1.399 & 1.2.E-04 & 2.9.E-04 & 0.27 \\
\hline ORZA9P & Unfavorable & 1.290 & 4.2.E-03 & 7.7.E-03 & 0.42 \\
\hline OR51E2 & Unfavorable & 1.242 & 1.3.E-05 & 3.7.E-05 & 0.27 \\
\hline ORAN2 & Favorable & 0.819 & 3.6.E-08 & 1.5.E-07 & 0.24 \\
\hline OR2113 & Favorable & 0.760 & 1.4.E-12 & 1.5.E-E-11 & 0.68 \\
\hline OR1411 & Favorable & 0.734 & 4.2.E-E5 & 1.0.E-E-04 & 0.82 \\
\hline ORZA1 & Favorable & 0.731 & 2.1.E-E2 & 3.6.E- -2 & 0.07 \\
\hline ORIF1 & Favorable & 0.728 & 1.6.E- -05 & 4.2.2. -05 & \\
\hline OR2L2 & Favorable & 0.670 & 2.3..E-E6 & 8.2.E-06 & \\
\hline ORAN4 & Favorable & 0.647 & 2.0.E-10 & 1.6.E-09 & 0.37 \\
\hline OR52E2 & Favorable & 0.639 & 2.3..E- 03 & 4.5.E- -03 & 0.44 \\
\hline OR52N4 & Favorable & 0.636 & 8.3.E- -07 & 3.2.E-06 & 0.17 \\
\hline ORAN3P & Favorable & 0.615 & 1.0.E-05 & 3.1.E-05 & 0.64 \\
\hline OR2T8 & Favorable & 0.613 & 1.1.E-02 & 1.9.E-02 & 0.81 \\
\hline OR7Е37P & Favorable & 0.580 & 2.5.E-03 & 4.8.E-03 & 0.41 \\
\hline OR13A1 & Favorable & 0.573 & 4.8.E-E9 & 2.5.E- -08 & 0.25 \\
\hline OR1F2P & Favorable & 0.536 & 2.1.E-03 & 4.3.E-03 & 0.89 \\
\hline ОR2H2 & Favorable & 0.532 & 2.2.E-17 & 4.6.E-16 & \\
\hline OR2C1 & Favorable & 0.465 & 1.8.E-15 & & \\
\hline OR5K2 & Favorable & 0.458 & 6.4.E-10 & 4.5.E- -99 & 0.31 \\
\hline OR5K1 & Favorable & 0.132 & 2.7. E- -08 & 1.2.E-E7 & 0.22 \\
\hline
\end{tabular}

B

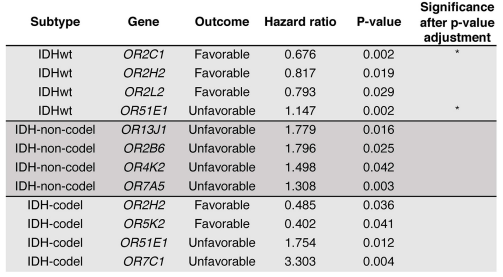

E

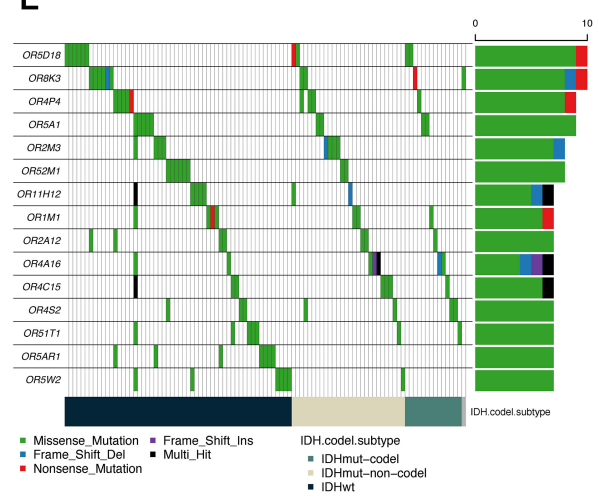

C
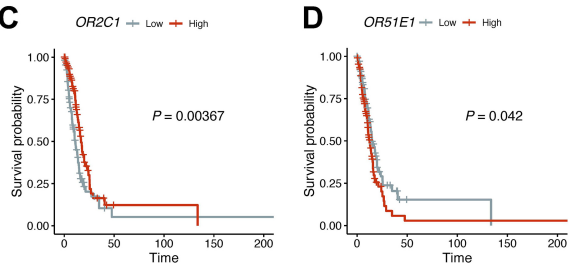

$\mathbf{F}$

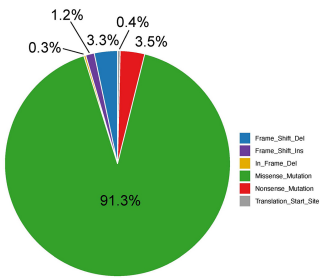

G

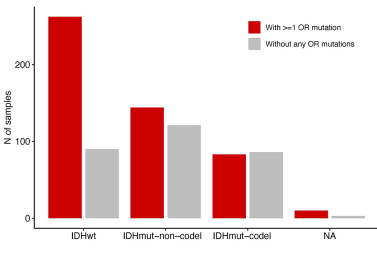

Fig. 2. Cox regression survival analysis of odorant receptor (OR) gene expression levels and somatic mutational profiling of OR genes in glioma. (A) A table describing univariate and multivariate Cox regression analysis of overall survival of OR genes in all glioma subtypes. Multivariate analysis was performed with age, IDH status, and histological grade. (B) A table describing OR genes that had expression levels significantly ( $\mathrm{P}<0.05$ before $\mathrm{P}$-value adjustment) associated with overall survival using the Cox proportional hazards regression model. An asterisk indicates the statistical significance after the P-value adjustment $(\mathrm{P}<0.05)$. (C, D) A Kaplan-Meir survival curve showing the survival difference between the OR2C1/Olfr15-high group and the OR2C1/Olfr15-low group (C) or the OR51E1/Olfr558-high group and the OR51E1/Olfr558-low group (D). A high group represents samples with expression levels higher than the median expression level, and the remainder of the samples were assigned as the low group. (E) The mutational landscape of the 15 most mutated OR genes. The bottom bar indicates the glioma subtype, and the right bars indicate the number of samples harboring mutations in each OR gene. (F) The variant type distribution of somatic mutations of OR genes in glioma. (G) Bar plots showing the number of samples that have mutations in at least one OR gene and samples without OR mutations according to glioma subtype. 
somatic mutation. Overall, 330 OR genes went through 1056 somatic mutations in the 499 samples, and of the 1056 mutations, $964(91.3 \%)$ were missense mutations (Fig. 2F). For IDHwt gliomas, 262 samples $(74.4 \%)$ possessed at least one OR mutation, while 144 of 265 (54.3\%) IDHmut-non-codel samples and 83 of 169 (49.1\%) IDHmut-codel samples had mutations in OR genes (Fig. 2G). Figure 2E summarizes the 15 most mutated OR genes in gliomas. OR5D18 (mouse Olfr1155) was the most frequently mutated OR gene and was detected in 10 samples. Of note, OR5AR1 (mouse Olfr1019) mutations were detected in seven IDHwt samples, but OR5AR1 (mouse Olfr1019) mutations were absent in IDHmut gliomas. Taking these findings together, we suggest that functionally validating mutations in OR genes will be key to understanding the biology of gliomas.

\section{CONCLUSION}

To date, the role of ORs in glioma has barely been investigated and is likely to be underestimated. Through a systematic analysis and review of olfactory GPCRs in glioma, we revealed that there is glioma subtype-specific expression of ORs such as OR51E1/Olfr558, OR51E2/Olfr78, OR4N2/Olfr733, OR2L13/ Olfr166, OR4N4, and OR4N3P. The existence of subtype-specific OR expression indicates that these ORs likely play roles in tumor formation and in the tumor microenvironment during glioma development or progression. In addition, identifying ORs associated with overall survival rates in glioma patients suggests that the expression level of ORs is a potential biomarker to predict glioma prognosis, and that these ORs should be investigated as therapeutic targets. To evaluate the oncogenic ability of ORs, an in-depth multi-omics analysis should be conducted with patient-derived resources and reliable translational models. Moreover, ORs are expressed in normal brain, and expression is up- and down-regulated in neurodegenerative diseases $(32,34)$. These observations demonstrate that ORs expressed in brain stromal cells and immune cells might form part of the glioma microenvironment and hence contribute to tumor progression. Therefore, single-cell RNA-sequencing analysis and reliable in vivo and/or in vitro models mimicking the tumor microenvironment are required to screen and functionally validate the cell type-specific expression of ORs.

\section{ACKNOWLEDGEMENTS}

This work was supported by grants from the National Research Foundation (2021R1A2C1009258 to J. Koo and 2021R1C1C1 004653 to H.J. Cho), the Bio \& Medical Technology Development Program (2020M3A9D3038435 to J. Koo), the Korean Mouse Phenotype Center (2019M3A9D5A01102797 to J. Koo).

\section{CONFLICTS OF INTEREST}

The authors have no conflicting interests.

\section{REFERENCES}

1. Schwartzbaum JA, Fisher JL, Aldape KD and Wrensch $M$ (2006) Epidemiology and molecular pathology of glioma. Nat Clin Pract Neurol 2, 494-503; quiz 491 p following 516

2. Wen PY and Kesari S (2008) Malignant gliomas in adults. N Engl J Med 359, 492-507

3. Stupp R, Mason WP, van den Bent MJ et al (2005) Radiotherapy plus concomitant and adjuvant temozolomide for glioblastoma. N Engl J Med 352, 987-996

4. Louis DN, Perry A, Wesseling P et al (2021) The 2021 WHO classification of tumors of the central nervous system: a summary. Neuro Oncol 23, 1231-1251

5. Brennan CW, Verhaak RG, McKenna A et al (2013) The somatic genomic landscape of glioblastoma. Cell 155, 462477

6. Ceccarelli M, Barthel FP, Malta TM et al (2016) Molecular profiling reveals biologically discrete subsets and pathways of progression in diffuse glioma. Cell 164, 550-563

7. Cancer Genome Atlas Research N, Brat DJ, Verhaak RG et al (2015) Comprehensive, integrative genomic analysis of diffuse lower-grade gliomas. N Engl J Med 372, 2481-2498

8. Sriram K and Insel PA (2018) G protein-coupled receptors as targets for approved drugs: how many targets and how many drugs? Mol Pharmacol 93, 251-258

9. Hauser AS, Attwood MM, Rask-Andersen M, Schioth HB and Gloriam DE (2017) Trends in GPCR drug discovery: new agents, targets and indications. Nat Rev Drug Discov 16, 829-842

10. Wu A, Maxwell R, Xia $Y$ et al (2019) Combination antiCXCR4 and anti-PD-1 immunotherapy provides survival benefit in glioblastoma through immune cell modulation of tumor microenvironment. J Neurooncol 143, 241-249

11. Zhou Y, Larsen PH, Hao C and Yong VW (2002) CXCR4 is a major chemokine receptor on glioma cells and mediates their survival. J Biol Chem 277, 49481-49487

12. Di Pizio A, Behrens M and Krautwurst D (2019) Beyond the flavour: the potential druggability of chemosensory $G$ protein-coupled receptors. Int J Mol Sci 20, 1402

13. Kang $N$ and Koo J (2012) Olfactory receptors in nonchemosensory tissues. BMB Rep 45, 612-622

14. Massberg D and Hatt $\mathrm{H}$ (2018) Human olfactory receptors: novel cellular functions outside of the nose. Physiol Rev 98, 1739-1763

15. Bjarnadottir TK, Gloriam DE, Hellstrand SH, Kristiansson $H$, Fredriksson R and Schioth HB (2006) Comprehensive repertoire and phylogenetic analysis of the $\mathrm{G}$ protein-coupled receptors in human and mouse. Genomics 88, 263273

16. Byrne KF, Pal A, Curtin JF, Stephens JC and Kinsella GK (2021) G-protein-coupled receptors as therapeutic targets for glioblastoma. Drug Discov Today 26, 2858-2870

17. HGNC Database, HUGO Gene Nomenclature Committee (HGNC), European Molecular Biology Laboratory, European Bioinformatics Institute (EMBL-EBI), Wellcome Genome Campus, Hinxton, Cambridge CB10 1SD, United Kingdom www.genenames.org. November, 2021

18. Hamm HE (1998) The many faces of G protein signaling. J 
Biol Chem 273, 669-672

19. liri T, Farfel Z and Bourne HR (1998) G-protein diseases furnish a model for the turn-on switch. Nature 394, 35-38

20. Fredriksson R, Lagerstrom MC, Lundin LG and Schioth HB (2003) The G-protein-coupled receptors in the human genome form five main families. Phylogenetic analysis, paralogon groups, and fingerprints. Mol Pharmacol 63, 1256-1272

21. Nordstrom KJ, Sallman Almen $M$, Edstam MM, Fredriksson $\mathrm{R}$ and Schioth HB (2011) Independent HHsearch, Needleman-Wunsch-based, and motif analyses reveal the overall hierarchy for most of the G protein-coupled receptor families. Mol Biol Evol 28, 2471-2480

22. Regard JB, Sato IT and Coughlin SR (2008) Anatomical profiling of $\mathrm{G}$ protein-coupled receptor expression. Cell 135, 561-571

23. Hakak Y, Shrestha D, Goegel MC, Behan DP and Chalmers DT (2003) Global analysis of G-protein-coupled receptor signaling in human tissues. FEBS Lett 550, 11-17

24. Conzelmann S, Levai O, Bode B et al (2000) A novel brain receptor is expressed in a distinct population of olfactory sensory neurons. Eur J Neurosci 12, 3926-3934

25. Otaki JM, Yamamoto H and Firestein S (2004) Odorant receptor expression in the mouse cerebral cortex. J Neurobiol 58, 315-327

26. Raming K, Konzelmann S and Breer H (1998) Identification of a novel G-protein coupled receptor expressed in distinct brain regions and a defined olfactory zone. Recept Channels 6, 141-151

27. Weber M, Pehl U, Breer H and Strotmann J (2002) Olfactory receptor expressed in ganglia of the autonomic nervous system. J Neurosci Res 68, 176-184

28. Feldmesser E, Olender T, Khen M, Yanai I, Ophir R and Lancet D (2006) Widespread ectopic expression of olfactory receptor genes. BMC Genomics 7, 121

29. Flegel C, Manteniotis S, Osthold S, Hatt $\mathrm{H}$ and Gisselmann G (2013) Expression profile of ectopic olfactory receptors determined by deep sequencing. PLoS One 8 , e55368

30. Zhang X, Rogers M, Tian H et al (2004) High-throughput microarray detection of olfactory receptor gene expression in the mouse. Proc Natl Acad Sci U S A 101, 14168-14173

31. Gaudel F, Stephan D, Landel V, Sicard G, Feron F and Guiraudie-Capraz G (2019) Expression of the cerebral olfactory receptors Olfr110/111 and Olfr544 is altered during aging and in Alzheimer's disease-like mice. Mol Neurobiol 56, 2057-2072

32. Ansoleaga B, Garcia-Esparcia P, Llorens F, Moreno J, Aso E and Ferrer I (2013) Dysregulation of brain olfactory and taste receptors in AD, PSP and CJD, and AD-related model. Neuroscience 248, 369-382

33. Lin MS, Chiu IH and Lin CC (2021) Ultrarapid inflammation of the olfactory bulb after spinal cord injury: protective effects of the granulocyte colony-stimulating factor on early neurodegeneration in the brain. Front Aging Neurosci 13,701702

34. Garcia-Esparcia P, Schluter A, Carmona M et al (2013) Functional genomics reveals dysregulation of cortical olfactory receptors in Parkinson disease: novel putative chemoreceptors in the human brain. J Neuropathol Exp Neurol 72, 524-539

35. Ansoleaga B, Garcia-Esparcia P, Pinacho R, Haro JM,
Ramos B and Ferrer I (2015) Decrease in olfactory and taste receptor expression in the dorsolateral prefrontal cortex in chronic schizophrenia. J Psychiatr Res 60, 109-116

36. Grison A, Zucchelli S, Urzi A et al (2014) Mesencephalic dopaminergic neurons express a repertoire of olfactory receptors and respond to odorant-like molecules. BMC Genomics 15, 729

37. Cho T, Lee C, Lee N, Hong YR and Koo J (2019) Smallchain fatty acid activates astrocytic odorant receptor Olfr920. Biochem Biophys Res Commun 510, 383-387

38. Lee N, Jae Y, Kim M et al (2020) A pathogen-derived metabolite induces microglial activation via odorant receptors. FEBS J 287, 3841-3870

39. Lee N, Sa M, Hong YR, Lee CJ and Koo J (2018) Fatty acid increases cAMP-dependent lactate and MAO-B-dependent GABA production in mouse astrocytes by activating a galphas protein-coupled receptor. Exp Neurobiol 27, 365376

40. Ferrer I, Garcia-Esparcia P, Carmona M et al (2016) Olfactory receptors in non-chemosensory organs: the nervous system in health and disease. Front Aging Neurosci 8, 163

41. Insel PA, Sriram K, Wiley SZ et al (2018) GPCRomics: GPCR expression in cancer cells and tumors identifies new, potential biomarkers and therapeutic targets. Front Pharmacol 9, 431

42. Sriram K, Moyung K, Corriden R, Carter H and Insel PA (2019) GPCRs show widespread differential mRNA expression and frequent mutation and copy number variation in solid tumors. PLoS Biol 17, e3000434

43. $\mathrm{Wu} \mathrm{V}$, Yeerna $\mathrm{H}$, Nohata $\mathrm{N}$ et al (2019) Illuminating the Onco-GPCRome: novel G protein-coupled receptor-driven oncocrine networks and targets for cancer immunotherapy. J Biol Chem 294, 11062-11086

44. O'Hayre M, Vazquez-Prado J, Kufareva I et al (2013) The emerging mutational landscape of $\mathrm{G}$ proteins and G-proteincoupled receptors in cancer. Nat Rev Cancer 13, 412-424

45. Kalra S, Mittal A, Gupta K et al (2020) Analysis of singlecell transcriptomes links enrichment of olfactory receptors with cancer cell differentiation status and prognosis. Commun Biol 3, 506

46. Masjedi S, Zwiebel LJ and Giorgio TD (2019) Olfactory receptor gene abundance in invasive breast carcinoma. Sci Rep 9, 13736

47. Ranzani M, lyer V, Ibarra-Soria X et al (2017) Revisiting olfactory receptors as putative drivers of cancer. Wellcome Open Res 2, 9

48. Weber L, Massberg D, Becker C et al (2018) Olfactory receptors as biomarkers in human breast carcinoma tissues. Front Oncol 8, 33

49. Vadevoo SMP, Gunassekaran GR, Lee C et al (2021) The macrophage odorant receptor Olfr78 mediates the lactateinduced M2 phenotype of tumor-associated macrophages. Proc Natl Acad Sci U S A 118, e2102434118

50. Sa JK, Chang N, Lee HW et al (2020) Transcriptional regulatory networks of tumor-associated macrophages that drive malignancy in mesenchymal glioblastoma. Genome Biol 21, 216

51. Wang Q, Hu B, Hu X et al (2018) Tumor evolution of glioma-intrinsic gene expression subtypes associates with immunological changes in the microenvironment. Cancer 
Cell 33, 152

52. Zhao I, Chen AX, Gartrell RD et al (2019) Immune and genomic correlates of response to anti-PD-1 immunotherapy in glioblastoma. Nat Med 25, 462-469

53. Yuan Y, Qi P, Xiang W, Yanhui L, Yu L and Qing M (2020) Multi-omics analysis reveals novel subtypes and driver genes in glioblastoma. Front Genet 11, 565341

54. Wang Q, He Z and Chen Y (2019) Comprehensive analysis reveals a 4-gene signature in predicting response to temozolomide in low-grade glioma patients. Cancer Control 26, 1073274819855118

55. Zhao H, Du P, Peng R et al (2021) Long noncoding RNA OR7E156P/miR-143/HIF1A axis modulates the malignant behaviors of glioma cell and tumor growth in mice. Front Oncol 11, 690213

56. Hanchate NK, Kondoh K, Lu Z et al (2015) Single-cell transcriptomics reveals receptor transformations during olfactory neurogenesis. Science 350, 1251-1255

57. Serizawa S, Miyamichi K and Sakano H (2005) Negative feedback regulation ensures the one neuron-one receptor rule in the mouse olfactory system. Chem Senses 30 Suppl
1, i99-i100

58. Pappula AL, Rasheed S, Mirzaei G, Petreaca RC and Bouley RA (2021) A genome-wide profiling of glioma patients with an IDH1 mutation using the catalogue of somatic mutations in cancer database. Cancers (Basel) 13, 4299

59. Aquilanti E, Miller J, Santagata S, Cahill DP and Brastianos PK (2018) Updates in prognostic markers for gliomas. Neuro Oncol 20, vii17-vii26

60. Cui T, Tsolakis AV, Li SC et al (2013) Olfactory receptor 51E1 protein as a potential novel tissue biomarker for small intestine neuroendocrine carcinomas. Eur J Endocrinol 168, 253-261

61. Jimenez RC, Casajuana-Martin N, Garcia-Recio A et al (2021) The mutational landscape of human olfactory G protein-coupled receptors. BMC Biol 19, 21

62. Trimmer C, Keller A, Murphy NR et al (2019) Genetic variation across the human olfactory receptor repertoire alters odor perception. Proc Natl Acad Sci U S A 116, 9475-9480 